\title{
Índice térmico ambiental de produtividade para frangos de corte
}

\author{
Carlos M. Medeiros ${ }^{1}$, Fernando da C. Baêta ${ }^{2}$, Rita F. M. de Oliveira ${ }^{3}$,
} Ilda de F. F. Tinôco ${ }^{2}$, Luiz F. T. Albino ${ }^{3}$ \& Paulo R. Cecon ${ }^{4}$

\begin{abstract}
DEAS/UFAM. CEP 69077-000, Manaus, AM. Fone (92) 3647-4042. E-mail: cmedeiros@ufam.edu.br 2 DEA/UFV, CEP 36571-000, Viçosa, MG. Fone (31) 3899-2105. E-mail: baeta@ufv.br; iftinoco@ufv.br 3 DZO/UFV. Fone: (31) 3899-3322. E-mail: flavia@ufv.br; lalbino@ufv.br

4 DPI/UFV. Fone: (31) 3899-1781. E-mail: cecon@dpi.ufv.br
\end{abstract}

Protocolo 80 - 7/6/2002 - Aprovado em 14/5/2005

\begin{abstract}
Resumo: Com o objetivo de desenvolver um índice térmico ambiental de produtividade para frangos de corte - IAPfc, que reúna, em um único valor, os efeitos da temperatura, umidade e velocidade do ar, conduziu-se um experimento com frangos Avian Farm, machos, criados de 1 a 21 dias de idade, em galpões convencionais com condições climáticas naturais e dos 22 aos 42 dias (abate) em câmaras climáticas. Os tratamentos compreenderam combinações de 16, 20, 26, 32 e $36{ }^{\circ} \mathrm{C}$ de temperatura (t), com $20,34,55,76$ e $90 \%$ de umidade relativa (u) e $0 ; 0,6 ; 1,5$; 2,4 e 3,0 $\mathrm{m} \mathrm{s}^{-1}$ de velocidade do ar (v), sendo estas combinações feitas com base no delineamento composto central rotacional da metodologia de superfície de resposta. As dietas experimentais atenderam às exigências nutricionais, de acordo com cada fase de criação, e para todos os tratamentos. Os parâmetros avaliados foram: ganho de peso, consumo de ração diário, temperatura retal e freqüência respiratória, obtendo-se o modelo estatístico IAPfc $=45,6026-2,31072 \mathrm{t}$ $0,368331 u+9,70922 v+0,0549243 t^{2}+0,0012183 u^{2}+0,66329 v^{2}+0,0128968$ tu - 0,300928 tv - 0,0595245 uv, baseado no desempenho animal e em parâmetros fisiológicos.
\end{abstract}

Palavras-chave: avicultura, ambiência avícola, conforto térmico

\section{Environmental thermal index of productivity for broiler chickens}

\begin{abstract}
In this study, the influence of temperature, relative humidity and air velocity in the development process of Avian Farm male chickens was analyzed in order to develop an environmental thermal index for productivity of broiler chickens - IAPfc. The chickens were first raised from 1 to 21 days of age in a conventional poultry-shed under natural conditions. Between $22^{\text {nd }}$ and $42^{\text {nd }}$ day (slaughter) the chickens were kept in climatic chambers. The treatments consisted of combination of levels of variables: temperature (t) $16,20,26,32$ and $36{ }^{\circ} \mathrm{C}$; relative humidity (u) 20,34, 55, 76 and $90 \%$, and air velocity (v) $0 ; 0.6 ; 1.5 ; 2.4$ and $3.0 \mathrm{~m} \mathrm{~s}^{-1}$, according to the central rotatable composite design of the response surface methodology. The experimental diets attained the suggested requirements of each development phase. The diet composition was the same in all treatments. The analyzed parameters were: daily weight gain, feed intake, rectal temperature and respiratory rate. The statistical model obtained was IAPfC $=45.6026-2.31072 \mathrm{t}$ $0.368331 u+9.70922 v+0.0549243 t^{2}+0.0012183 u^{2}+0.66329 v^{2}+0.0128968 t u-$ $0.300928 \mathrm{tv}-0.0595245 \mathrm{uv}$, which is based on the animal performance and physiological parameters.
\end{abstract}

Key words: aviculture, poultry environment, thermal comfort

\section{INTRODUÇÃO}

Desde o início do século XX, o homem tem tentado quantificar o ambiente térmico animal, utilizando correlações nas quais são empregadas as variáveis: temperatura, umidade, velocidade do ar e radiação. Em alguns casos, também são consideradas outras variáveis, como a taxa metabólica, o tipo de isolamento, entre outros. 
Esses estudos tiveram início quando Houghten \& Yaglou (1923) propuseram o Índice de Temperatura Efetiva - ITE, baseado na temperatura, umidade e velocidade do ar, usando humanos para comparar sensações térmicas instantâneas, experimentadas em diferentes ambientes.

Do mesmo modo, propuseram-se outros índices, como: o P4SR (Predicted Four Hour Sweat Rate) estimativa da taxa de suor por quatro horas, McArdle (1947); Índice de Estresse Calórico, Belding \& Hatch (1955); Índice de Desconforto, mais tarde chamado Índice de Temperatura e Umidade - ITU, Thom (1959); Índice de Humiture, Hevener (1959) e o Índice de Temperatura Aparente, Steadman (1979); contudo, os índices mencionados foram desenvolvidos especificamente para seres humanos. Buffington et al. (1981), trabalhando com vacas leiteiras propuseram, com base no Índice de Temperatura e Umidade, o Índice de Temperatura de Globo Negro e Umidade - ITGU; Rosenberg et al. (1983), propuseram o Índice de Temperatura Baixa e Vento - ITBV e, a partir de estudos em câmaras climáticas, Baêta (1985) propôs o Índice de Temperatura Equivalente, para gado de leite, os índices são apresentados na Tabela 1.

Desses índices, o mais empregado até a década de 80 para avaliar o ambiente térmico animal, foi o ITU, na década de 90 , o ITGU; sabe-se, porém, que a intensidade de participação de cada variável térmica ambiental na composição do índice é diferente para cada espécie animal, em razão de diferenças fisiológicas correspondentes. O ITU e o ITGU podem ser usados para predizer a qualidade de um ambiente térmico para a ave, entretanto com baixa sensibilidade, uma vez que no seu desenvolvimento não se levou em conta a resposta animal. Deste modo, objetivou-se, com o presente estudo, estabelecer um índice térmico ambiental específico para frangos de corte, com base na temperatura, umidade e velocidade do ar, que permita quantificar o ambiente térmico, com base no desempenho animal.

\section{MATERIAL E MÉTODOS}

O experimento foi desenvolvido nos meses de maio, junho e julho de 2000, em câmaras climáticas do Laboratório de Bioclimatologia Animal do Departamento de Zootecnia da Universidade Federal de Viçosa, quando se utilizaram 960 pintos de um dia, machos, Avian Farm, criados em galpão convencional com condições climáticas naturais, até os 21 dias de idade; a partir dos 22 dias até a época do abate, aos 42 dias, os frangos foram alojados em câmaras climáticas, onde receberam água e ração à vontade.

Neste tipo de experimento, Box \& Wilson (1951), Box (1954), Cochran \& Cox (1957), Henika (1972) e Giovani (1983) afirmam que o delineamento composto central rotacional da Superfície de Resposta é o método de análise de resultados que proporciona um número maior de informações e o modelo mais adequado é a equação polinomial generalizada, descrita pela série de Taylor que, para três variáveis e para o segundo grau, pode ser escrita como:

Tabela 1. Autores e seus respectivos índices ambientais

\begin{tabular}{|c|c|c|}
\hline Autor & Índice & Descrição das Variáveis \\
\hline McArdle (1947) & $\mathrm{P} 4 \mathrm{SR}=\mathrm{B} 4 \mathrm{SR}+0,25$ & $\begin{array}{l}\mathrm{P} 4 \mathrm{SR}=\text { estimativa da taxa de suor } \\
\mathrm{B} 4 \mathrm{SR}=\text { taxa básica de suor em } 4 \text { horas, } \mathrm{L}\end{array}$ \\
\hline Belding \& Hatch (1955) & $\mathrm{HSI}=100($ Ereq/Emax $)$ & $\begin{array}{l}\mathrm{HSI}=\text { índice de estresse calórico } \\
\text { Ereq = fluxo de calor requerido, } \mathrm{W} \mathrm{m}^{-2} \\
\text { Emax = fluxo de calor máximo, } \mathrm{W} \mathrm{m}^{-2}\end{array}$ \\
\hline Hevener (1959) & $\mathrm{H}=\mathrm{t}+(\mathrm{e}-2)$ & $\begin{array}{l}\mathrm{H}=\text { índice de umidade } \\
\mathrm{t}=\text { temperatura do } \mathrm{ar},{ }^{\circ} \mathrm{F} \\
\mathrm{e}=\text { pressão de vapor, } \mathrm{mb} .\end{array}$ \\
\hline Thom (1959) & $\mathrm{ITU}=0,72(\mathrm{tb}+\mathrm{tbu})+40,6$ & $\begin{array}{l}\text { ITU = índice de temperatura e umidade } \\
\text { tb = temperatura de bulbo seco, }{ }^{\circ} \mathrm{C} \\
\text { tbu = temperatura de bulbo úmido, }{ }^{\circ} \mathrm{C}\end{array}$ \\
\hline Steadman (1979) & $\begin{array}{l}\text { ITE }=-42,379+2,04901523 \mathrm{t}+10,14333127 \mathrm{r}-0,22475541 \mathrm{tr}- \\
6,83783 \times 10^{-3} \mathrm{t}^{2}-5,481717 \times 10^{-2} \mathrm{r}^{2}+1,22874 \times 10^{-3} \mathrm{t}^{2} \mathrm{r}+ \\
8,5282 \times 10^{-4} \cdot \mathrm{tr}^{2}-1,99 \times 10^{-6} \mathrm{t}^{2} \mathrm{r}^{2}\end{array}$ & $\begin{array}{l}\text { ITE }=\text { índice de temperatura aparente } \\
\mathrm{t}=\text { temperatura de bulbo seco, }{ }^{\circ} \mathrm{C} \\
\mathrm{r}=\text { umidade relativa, } \%\end{array}$ \\
\hline Buffington et al. (1981) & $\mathrm{ITGU}=\operatorname{tgn}+0,36$ tpo $+41,5$ & $\begin{array}{l}\text { ITGU = índice de temperatura de globo e } \\
\text { umidade } \\
\text { tgn }=\text { temperatura de globo negro, }{ }^{\circ} \mathrm{C} \\
\text { tpo }=\text { temperatura ponto de orvalho, }{ }^{\circ} \mathrm{C}\end{array}$ \\
\hline Rosenberg et al. (1983) & $\mathrm{ITBV}=31,68738-4,284707 \times 10^{-2} \mathrm{Q}-1,051564 \times 10^{-6} \mathrm{Q}^{2}$ & $\begin{array}{l}\text { ITBV }=\text { índice de temperature baixa } \\
\mathrm{Q}=\left(10,45+10 \mathrm{v}^{0,5} \text {-ta }\right)(33-\text { ta }) \\
\text { ta }=\text { temperatura do } \mathrm{ar},{ }^{\circ} \mathrm{C} \\
\mathrm{v}=\text { velocidade do ar, } \mathrm{ms}^{-1}\end{array}$ \\
\hline Baêta (1985) & $\begin{array}{l}\text { ITE }=27,88-0,456 \mathrm{t}+0,0100754 \mathrm{t}^{2}-0,4905 \mathrm{ur}+0,00088 \mathrm{ur}^{2}+ \\
1,1507 \mathrm{v}-0,126447 \mathrm{v}^{2}+0,019876 \text { tur }-0,046313 \mathrm{tv}\end{array}$ & $\begin{array}{l}\text { ITE }=\text { índice de temperatura equivalente } \\
\mathrm{T}=\text { temperatura, }{ }^{\circ} \mathrm{C} \\
\text { ur }=\text { umidade relativa, } \% \\
\mathrm{v}=\text { velocidade do ar, } \mathrm{m} \mathrm{s}^{-1}\end{array}$ \\
\hline
\end{tabular}


$\eta_{\mathrm{i}}=\beta_{0}+($ ponto central $)$

$$
\begin{aligned}
& \beta_{1} \mathrm{t}+\beta_{2} \mathrm{u}+\beta_{3} \mathrm{v}+(\text { efeitos lineares }) \\
& \beta_{11} \mathrm{t}^{2}+\beta_{22} \mathrm{u}^{2}+\beta_{33} \mathrm{v}^{2}+(\text { efeitos de segunda ordem) } \\
& \beta_{12} \mathrm{tu}+\beta_{13} \mathrm{tv}+\beta_{23} \mathrm{uv} \text { (interação) }
\end{aligned}
$$

em que:

$\eta_{i-}$ resposta medida no tratamento $i$,

$\beta_{\mathrm{o}}, \beta_{1}, \beta_{2}, \ldots$ - parâmetros da equação,

$\mathrm{t}$ - temperatura do ar, ${ }^{\circ} \mathrm{C}$,

$\mathrm{u}$ - umidade relativa do ar, $\%$, e

$\mathrm{v}$ - velocidade do ar, $\mathrm{m} \mathrm{s}^{-1}$

Os valores das variáveis naturais foram $16,20,26,32$ e $36^{\circ} \mathrm{C}$ de temperatura; 20,34, 55, 76 e $90 \%$ de umidade relativa e $0 ; 0,6$; 1,$5 ; 2,4$ e $3,0 \mathrm{~m} \mathrm{~s}^{-1}$ de velocidade do ar. Os tratamentos tiveram como base o delineamento composto central rotacional, cuja codificação $-1,682 ;-1 ; 0 ; 1 ; 1,682$ estabeleceu a combinação entre as variáveis naturais, totalizando 15 tratamentos e 5 repetições do ponto central $(0,0,0)$ como mostrado na Tabela 2 .

\begin{tabular}{|c|c|c|c|c|c|c|}
\hline \multirow[b]{2}{*}{ Tratamento } & \multicolumn{3}{|c|}{ Variáveis Naturais } & \multicolumn{3}{|c|}{ Variáveis Codificadas } \\
\hline & $\begin{array}{c}\mathrm{t} \\
\left({ }^{\circ} \mathrm{C}\right)\end{array}$ & $\begin{array}{c}u \\
(\%)\end{array}$ & $\begin{array}{c}\mathrm{v} \\
\left(\mathrm{m} \mathrm{s}^{-1}\right)\end{array}$ & $\mathrm{t}$ & $\mathrm{u}$ & $\mathrm{v}$ \\
\hline 1 & 20 & 34 & 0,6 & -1 & -1 & -1 \\
\hline 2 & 20 & 34 & 2,4 & 1 & -1 & -1 \\
\hline 3 & 20 & 76 & 0,6 & -1 & 1 & -1 \\
\hline 4 & 20 & 76 & 2,4 & 1 & 1 & -1 \\
\hline 5 & 32 & 34 & 0,6 & -1 & -1 & 1 \\
\hline 6 & 32 & 34 & 2,4 & 1 & -1 & 1 \\
\hline 7 & 32 & 76 & 0,6 & -1 & 1 & 1 \\
\hline 8 & 32 & 76 & 2,4 & 1 & 1 & 1 \\
\hline 9 & 16 & 55 & 1,5 & $-1,682$ & 0 & 0 \\
\hline 10 & 36 & 55 & 1,5 & 1,682 & 0 & 0 \\
\hline 11 & 26 & 20 & 1,5 & 0 & $-1,682$ & 0 \\
\hline 12 & 26 & 90 & 1,5 & 0 & 1,682 & 0 \\
\hline 13 & 26 & 55 & 0,0 & 0 & 0 & $-1,682$ \\
\hline 14 & 26 & 55 & 3,0 & 0 & 0 & 1,682 \\
\hline 15 & 26 & 55 & 1,5 & 0 & 0 & 0 \\
\hline 16 & 26 & 55 & 1,5 & 0 & 0 & 0 \\
\hline 17 & 26 & 55 & 1,5 & 0 & 0 & 0 \\
\hline 18 & 26 & 55 & 1,5 & 0 & 0 & 0 \\
\hline 19 & 26 & 55 & 1,5 & 0 & 0 & 0 \\
\hline 20 & 26 & 55 & 1,5 & 0 & 0 & 0 \\
\hline
\end{tabular}

Tabela 2. Tratamentos experimentais, variáveis naturais e codificadas

A movimentação do ar no interior das câmaras climáticas cujas dimensões eram 5,70 x 2,85 x 2,10 m, respectivamente, largura, comprimento e altura, foi feita com o uso de ventiladores axiais, com vazão de $3,33 \mathrm{~m}^{3} \mathrm{~s}^{-1}$, equipados com difusores de papelão, para distribuir uniformemente, o ar, na direção das aves.

Antes de serem alojadas nas câmaras climáticas, as aves foram pesadas, e descartadas aquelas com alto desvio-padrão em torno da média, uniformizando o peso inicial. Cada tratamento foi composto de 48 aves, distribuídas em 12 gaiolas, cada gaiola apresentava as dimensões de $0,85 \times 0,85 \times 0,40 \mathrm{~m}$, agrupadas em baterias de 3 gaiolas por andar, sendo ao todo 4 andares dentro de cada câmara.

As respostas determinadas dos frangos foram: ganho de peso, consumo de ração, temperatura retal e freqüência respiratória. Para a avaliação do ganho de peso, as aves foram pesadas no início do experimento ( 22 dias de idade) e no final do período experimental (42 dias de idade); A medida do consumo de ração por ave foi feita a partir da diferença entre o total fornecido (ração armazenada em recipientes plásticos com capacidade para $15 \mathrm{~kg}$ de ração) menos o total perdido (recolhido no chão) e as sobras. $\mathrm{O}$ valor total médio consumido por ave foi obtido por meio da divisão do total de ração consumida por 4 (número de aves por gaiola).

A determinação da temperatura retal e da freqüência respiratória dos animais foi feita em intervalos de seis em seis dias (28, 34 e 40 dias de idade), às nove horas da manhã, em uma ave por grupo, escolhida ao acaso. Na medição da temperatura retal utilizou-se termômetro de termistor, Digi-sensi, digital, com resolução de $\pm 0,1^{\circ} \mathrm{C}$, com o sensor (sonda número 400 ) introduzido no reto das aves, durante um minuto. Obtevese a freqüência respiratória a partir da contagem do número de movimentos abdominais, por um período de $3 \mathrm{~min}$; neste processo, utilizou-se um cronômetro digital, para registro do tempo.

A velocidade do ar foi aferida diariamente, no centro de cada gaiola, empregando-se anemômetro de hélice digital, com resolução de $0,1 \mathrm{~ms}^{-1}$.

\section{RESULTADOS E DISCUSSÃO}

O ganho de peso diário (GPD), o consumo diário de ração (CDR), a temperatura retal (TR) e a freqüência respiratória (FR) em função das diferentes combinações de temperatura $(\mathrm{t})$, umidade (u) e velocidade do ar (v), são apresentados na Tabela 3.

Como pressuposto, estabeleceu-se que o índice adequado a esta situação seria aquele em que a comparação entre as diversas combinações de temperatura, umidade relativa e velocidade do ar, fossem feitas com base nas respostas fisiológicas e de produtividade das aves. Então, se dois ambientes térmicos caracterizados por combinações com diferentes valores das variáveis consideradas conduzem a uma mesma resposta fisiológica, ou de produtividade, é porque eles têm a mesma qualidade ambiental.

Deste modo e a fim de gerar os valores básicos de comparação referentes a ganho de peso diário, consumo de ração diário, temperatura retal e freqüência respiratória, tomaram-se como referência os valores de temperatura, associados a um valor de umidade relativa, que correspondeu à melhor resposta animal na ausência da velocidade do ar, com base na escala Celsius. Para tal, partiu-se dos modelos estatísticos desenvolvidos por Medeiros (2001) nos quais foram estimadas as melhores respostas animais em função da temperatura, umidade relativa e velocidade do ar, para as condições experimentais (Tabela 4).

Observa-se, na Tabela 4, que a umidade relativa de 90\% conduziu aos melhores resultados de produtividade para os animais, quando combinada com baixas temperaturas, ao passo que umidades relativas de $20 \%$ conduziram aos melhores 
Tabela 3. Respostas dos frangos de corte na fase de 22 aos 42 dias de idade, em função da temperatura, umidade relativa e velocidade do ar

\begin{tabular}{cccccccc}
\hline $\mathrm{t}$ & $\mathrm{u}$ & $\mathrm{v}$ & & \multicolumn{3}{c}{ Resposta dos frangos } \\
\cline { 1 - 3 }$\left({ }^{\circ} \mathrm{C}\right)$ & $(\%)$ & $\left(\mathrm{m} \mathrm{s}^{-1}\right)$ & $\mathrm{GPD}(\mathrm{g})$ & $\mathrm{CRD}(\mathrm{g})$ & $\mathrm{TR}\left({ }^{\circ} \mathrm{C}\right) \mathrm{FR}\left(\mathrm{resp} \mathrm{min}^{-1}\right)$ \\
\hline 20 & 34 & 0,6 & & 74,44 & 146,47 & 40,9 & 45 \\
20 & 34 & 2,4 & 72,73 & 148,43 & 41,0 & 47 \\
20 & 76 & 0,6 & 78,43 & 147,06 & 41,0 & 38 \\
20 & 76 & 2,4 & & 76,16 & 147,07 & 41,0 & 44 \\
32 & 34 & 0,6 & 60,33 & 119,28 & 41,8 & 77 \\
32 & 34 & 2,4 & 64,97 & 126,57 & 41,7 & 71 \\
32 & 76 & 0,6 & 56,16 & 112,87 & 42,3 & 98 \\
32 & 76 & 2,4 & 60,25 & 118,22 & 42,1 & 96 \\
16 & 55 & 1,5 & 60,40 & 138,13 & 41,1 & 50 \\
36 & 55 & 1,5 & 35,37 & 91,42 & 42,8 & 120 \\
26 & 20 & 1,5 & 79,11 & 146,56 & 41,0 & 45 \\
26 & 90 & 1,5 & 78,50 & 140,09 & 41,4 & 60 \\
26 & 55 & 0 & 78,12 & 140,15 & 41,2 & 52 \\
26 & 55 & 3,0 & 80,10 & 146,24 & 41,1 & 52 \\
26 & 55 & 1,5 & 76,92 & 140,25 & 41,1 & 44 \\
26 & 55 & 1,5 & 76,92 & 140,25 & 41,1 & 44 \\
26 & 55 & 1,5 & 76,92 & 140,25 & 41,1 & 44 \\
26 & 55 & 1,5 & 76,92 & 140,25 & 41,1 & 44 \\
26 & 55 & 1,5 & 76,92 & 140,25 & 41,1 & 44 \\
26 & 55 & 1,5 & 76,92 & 140,25 & 41,1 & 44 \\
\hline & & & & & & \\
\hline
\end{tabular}

Tabela 4. Valores de referência $(\mathrm{t}, \mathrm{u}, \mathrm{v})$ para as melhores respostas dos frangos de corte, na fase de 22 aos 42 dias de idade

\begin{tabular}{ccccccc}
\hline $\mathrm{t}$ & $\mathrm{u}$ & \multicolumn{2}{c}{$\mathrm{v}$} & \multicolumn{4}{c}{ Parâmetros estimados } \\
\cline { 1 - 4 } \cline { 1 - 2 }$\left({ }^{\circ} \mathrm{C}\right)$ & $(\%)$ & $\left(\mathrm{m} \mathrm{s}^{-1}\right)$ & $\mathrm{GPD}(\mathrm{g})$ & $\mathrm{CRD}(\mathrm{g})$ & $\mathrm{TR}\left({ }^{\circ} \mathrm{C}\right)$ & $\mathrm{FR}\left(\right.$ resp $\left.\mathrm{min}^{-1}\right)$ \\
\hline 16 & 90 & 0 & 73,65 & 147,79 & 41,2 & 46 \\
17 & 90 & 0 & 76,91 & 149,44 & 41,1 & 44 \\
18 & 90 & 0 & 79,58 & 150,57 & 41,1 & 43 \\
19 & 90 & 0 & 81,68 & 151,20 & 41,1 & 43 \\
20 & 90 & 0 & 83,19 & 151,33 & 41,1 & 44 \\
21 & 90 & 0 & 84,13 & 150,94 & 41,1 & 45 \\
22 & 90 & 0 & 84,48 & 150,04 & 41,2 & 48 \\
23 & 90 & 0 & 84,26 & 148,63 & 41,2 & 51 \\
24 & 90 & 0 & 83,45 & 146,71 & 41,3 & 55 \\
25 & 90 & 0 & 82,06 & 144,29 & 41,4 & 60 \\
26 & 90 & 0 & 80,09 & 141,35 & 41,5 & 65 \\
27 & 20 & 0 & 78,51 & 142,64 & 41,2 & 57 \\
28 & 20 & 0 & 76,51 & 139,65 & 41,3 & 60 \\
29 & 20 & 0 & 73,93 & 136,16 & 41,4 & 64 \\
30 & 20 & 0 & 70,77 & 132,16 & 41,5 & 69 \\
31 & 20 & 0 & 67,03 & 127,65 & 41,6 & 75 \\
32 & 20 & 0 & 62,71 & 122,62 & 41,8 & 81 \\
33 & 20 & 0 & 57,81 & 117,09 & 42,0 & 89 \\
34 & 20 & 0 & 52,33 & 111,05 & 42,2 & 97 \\
35 & 20 & 0 & 46,27 & 104,50 & 42,4 & 106 \\
36 & 20 & 0 & 39,63 & 97,44 & 42,6 & 116 \\
\hline
\end{tabular}

resultados quando combinadas com altas temperaturas, na ausência da velocidade do ar.
A partir da Tabela 4, estabeleceram-se correlações entre os valores de temperatura e as diversas respostas animais, cujos parâmetros estimados foram denominados temperatura de referência (Tref). As equações correspondentes para cada resposta animal foram:

$$
\begin{gathered}
\operatorname{Tref}(\mathrm{GPD})=232,512-5,93168 \mathrm{GPD}+0,0406476 \mathrm{GPD}^{2} \\
\mathrm{R}^{2}=0,98 \\
\operatorname{Tref}(\mathrm{CRD})=-0,553415+0,766402 \mathrm{CRD}-0,0040659 \mathrm{CRD}^{2} \\
\mathrm{R}^{2}=0,98 \\
\operatorname{Tref}(\mathrm{TR})=-17004,3+805,503 \mathrm{TR}-9,51945 \mathrm{TR}^{2} \\
\mathrm{R}^{2}=0,82 \\
\operatorname{Tref}(\mathrm{FR})=-13,0572+0,932671 \mathrm{FR}-0,00449971 \mathrm{FR}^{2} \\
\mathrm{R}^{2}=0,97
\end{gathered}
$$

Assim, a Tref(GPD) é a condição térmica do ambiente, expressa em termos de $\left({ }^{\circ} \mathrm{C}\right)$ que corresponde ao ganho de peso diário (GPD) em questão. Correlacionando-se as médias das temperaturas de referência do GPD, CRD, TR e FR, com os valores correspondentes de temperatura, umidade e velocidade do ar, obteve-se o Índice Térmico Ambiental de Produtividade para frango de corte, cuja expressão é dada por:

$$
\begin{gathered}
\text { IAPfc }=45,6026-2,31072 \mathrm{t}-0,368331 \mathrm{u}+9,70922 \mathrm{v}+ \\
0,0549243 \mathrm{t}^{2}+0,0012183 \mathrm{u}^{2}+0,66329 \mathrm{v}^{2}+ \\
0,012897 \mathrm{tu}-0,300928 \mathrm{tv}-0,0595245 \mathrm{uv} \quad \mathrm{R}^{2}=0,94
\end{gathered}
$$

Aplicando-se os valores de temperatura, umidade e velocidade do ar (Tabela 2) na equação do IAPfc e se correlacionando com os valores de GPD, CRD, TR eFR (Tabela 3), obtiveram-se as equações:

$$
\begin{gathered}
\mathrm{GPD}=-17,6849+8,31555 \mathrm{IAP}-0,182259 \mathrm{IAP}^{2} \\
\mathrm{R}^{2}=0,68 \\
\mathrm{CRD}=64,5181+7,72478 \mathrm{IAP}-0,185353 \mathrm{IAP}^{2} \\
\mathrm{R}^{2}=0,78 \\
\mathrm{TR}=44,2137-0,307612 \mathrm{IAP}+0,00731497 \mathrm{IAP}^{2} \\
\mathrm{R}^{2}=0,86 \\
\mathrm{FR}=192,682-14,008 \mathrm{IAP}+0,327686 \mathrm{IAP}^{2} \\
\mathrm{R}^{2}=0,87
\end{gathered}
$$

Defrontando-se os valores de GPD, CRD, TR e FR estimados por essas equações com os valores experimentais (Tabela 2), obtiveram-se as correlações 0,$89 ; 0,93 ; 0,96$ e 0,95 , respectivamente, conforme mostra a Figura 1.

Desta forma, estimaram-se os valores do ganho de peso diário - GPD, consumo diário de ração - $\mathrm{CRD}$, temperatura retal - TR e freqüência respiratória - FR, em função do IAPfc variando de 16 a 42 e se considerando que o maior GPD é de 77,16 $\mathrm{g} \mathrm{dia}^{-1}$ ave $^{-1}$; para um CRD de 144,14 $\mathrm{g} \mathrm{dia}^{-1}$ ave $^{-1}$, obteve-se a perda de desempenho animal, em termos de GPD e CRD.

Observando-se as variações de desempenho na Tabela 5, nota-se que os valores do índice entre 21 e 24 estão associados 

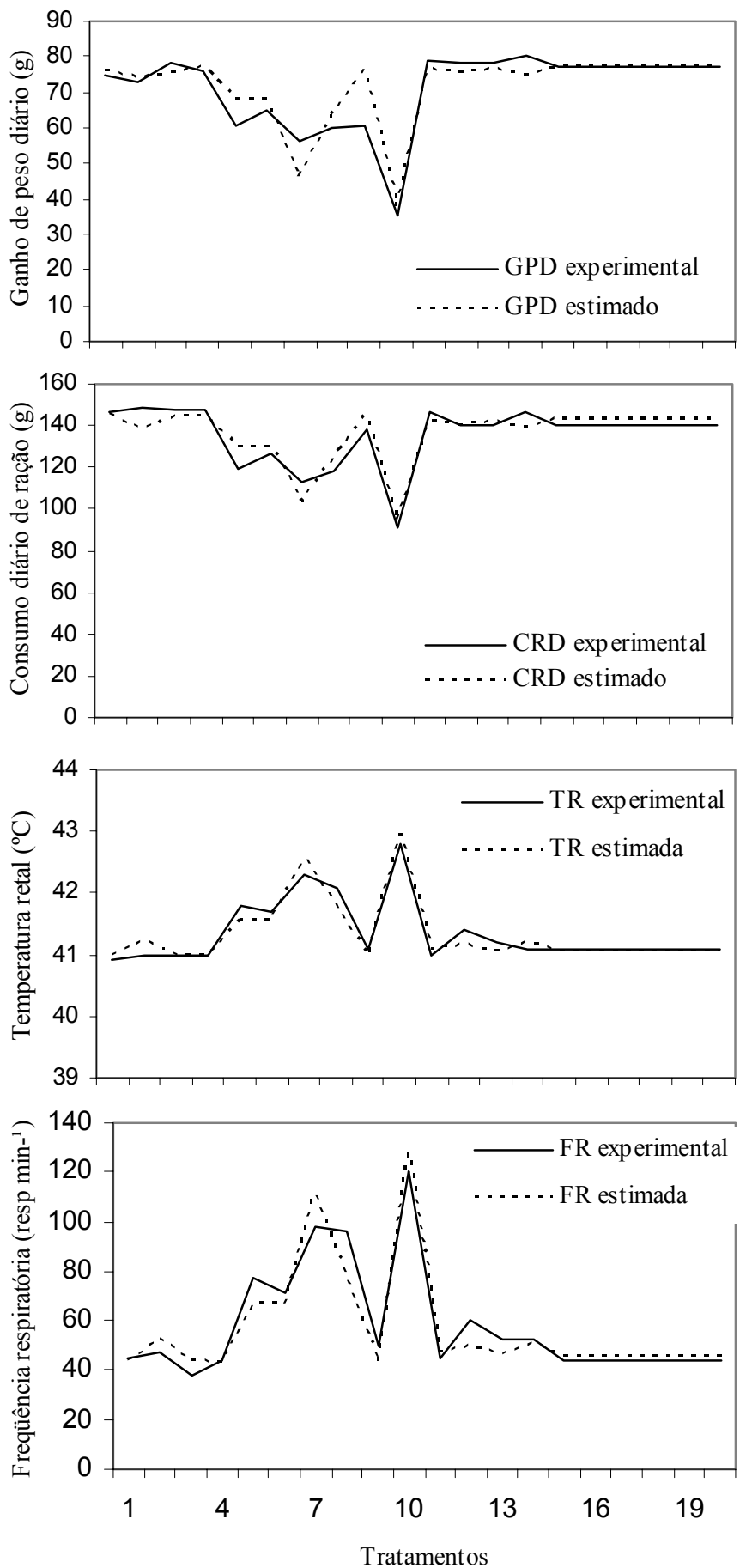

Figura 1. Valores de Ganho de Peso Diário (GPD), Consumo Diário de reação (CRD), Temperatura Retal (TR) e Frequência Respiratória (FR) experimentais e estimados

à máxima produtividade; para valores entre 25 e 27, registrouse perda de peso da ave, em torno de 1 a $5 \%$; para valores entre 28 e 30 , perda de 5 a $15 \%$; para valores entre 31 e 34, perda de 15 a $30 \%$ e, para valores acima de 35 , perda de 30 a $87 \%$.

Conseqüentemente, pode-se qualificar o ambiente climático através do IAPfc como sendo: confortável, quando o IAPfc varia de 21 a 24; moderadamente confortável, de 19 a 20 e 25 a 27; desconfortável, abaixo de 19 e de 28 a 30; extremamente desconfortável, de 31 a 34 e perigoso quando o IAPfc está igual ou acima de 35 .
Tabela 5. Valores médios estimados do GPD, CRD, TR e FR e variação do GPD e CRD, em função do IAPfc, para a fase de 22 aos 42 dias de idade

\begin{tabular}{|c|c|c|c|c|c|c|}
\hline \multirow[b]{2}{*}{ IAPfc } & \multicolumn{4}{|c|}{ Parâmetros estimados } & \multicolumn{2}{|c|}{ Variação } \\
\hline & $\begin{array}{l}\text { GPD } \\
(\mathrm{g})\end{array}$ & $\begin{array}{l}\text { CRD } \\
(\mathrm{g})\end{array}$ & $\begin{array}{c}\mathrm{TR} \\
\left({ }^{\circ} \mathrm{C}\right)\end{array}$ & $\begin{array}{c}\text { FR } \\
\left(\text { respmin }^{-1}\right)\end{array}$ & $\begin{array}{c}\text { GPD } \\
(\%)\end{array}$ & $\begin{array}{c}\text { CRD } \\
(\%)\end{array}$ \\
\hline 16 & 67,45 & 139,74 & 41,2 & 54 & $-12,6$ & $-3,1$ \\
\hline 17 & 71,01 & 142,27 & 41,1 & 49 & $-8,0$ & $-1,3$ \\
\hline 18 & 72,94 & 143,51 & 41,0 & 47 & $-5,5$ & $-0,4$ \\
\hline 19 & 74,52 & 144,38 & 41,0 & 45 & $-3,4$ & 0,2 \\
\hline 20 & 75,72 & 144,87 & 41,0 & 44 & $-1,8$ & 0,5 \\
\hline 21 & 76,57 & 145,00 & 41,0 & 43 & $-0,8$ & 0,6 \\
\hline 22 & 77,04 & 144,75 & 41,0 & 43 & $-0,2$ & 0,4 \\
\hline 23 & 77,16 & 144,14 & 41,0 & 44 & 0,0 & 0,0 \\
\hline 24 & 76,91 & 143,15 & 41,0 & 45 & $-0,3$ & $-0,7$ \\
\hline 25 & 76,29 & 141,79 & 41,1 & 47 & $-1,1$ & $-1,6$ \\
\hline 26 & 75,31 & 140,06 & 41,2 & 50 & $-2,4$ & $-2,8$ \\
\hline 27 & 73,97 & 137,96 & 41,2 & 53 & $-4,1$ & $-4,3$ \\
\hline 28 & 72,26 & 135,50 & 41,3 & 57 & $-5,7$ & $-6,0$ \\
\hline 29 & 70,19 & 132,65 & 41,4 & 62 & $-9,0$ & $-8,0$ \\
\hline 30 & 67,75 & 129,44 & 41,6 & 67 & $-12,2$ & $-10,2$ \\
\hline 31 & 64,95 & 125,86 & 41,7 & 73 & $-15,8$ & $-12,7$ \\
\hline 32 & 61,78 & 121,91 & 41,9 & 80 & $-19,9$ & $-15,4$ \\
\hline 33 & 58,25 & 117,59 & 42,0 & 87 & $-24,5$ & $-18,4$ \\
\hline 34 & 54,35 & 112,89 & 42,2 & 95 & $-29,7$ & $-21,7$ \\
\hline 35 & 50,09 & 107,83 & 42,4 & 104 & $-35,1$ & $-25,2$ \\
\hline 36 & 45,47 & 102,39 & 42,6 & 113 & $-41,1$ & $-29,0$ \\
\hline 38 & 35,12 & 90,41 & 43,1 & 134 & $-54,5$ & $-37,3$ \\
\hline 40 & 23,32 & 76,94 & 43,6 & 157 & $-69,8$ & $-46,6$ \\
\hline 42 & 10,06 & 62,00 & 44,2 & 182 & $-87,0$ & $-57,0$ \\
\hline
\end{tabular}

\section{CONCLUSÃO}

O índice térmico ambiental de produtividade para frango de corte (IAPfc) é um indicador de conforto e produção animal desenvolvido sob condições experimentais, relaciona desempenho animal e parâmetros fisiológicos dos frangos de 22 a 42 dias de idade com o ambiente.

\section{LITERATURA CITADA}

Baêta, F.C. Responses of lactating dairy cows to the combined effects of temperature, humidity and wind velocity in the warn season. Missouri: University of Missouri - Columbia, 1985. 218p. Ph.D thesis

Belding, H.S.; Hatch, T.F. Index for evaluating heat stress in terms of resulting physiological strain. Heating, Piping and Air Conditioning, Cleaveland, v.3, n.27, p.129-136, 1955.

Box, G.E.P. The exploitation and exploitation of response surfaces: some general conditions and examples. Biometrics, Texas, v.10, n.1, p.16-60, 1954.

Box, G.E.P.; Wilson, K.B. On the experimental attainment of optimum conditions. Journal of the Royal Statistic Society: series B (Statistical Methodology), Manchester, v.13, n.1, p.138,1951 . 
Buffington, D.E.; Collasso-arocho, A.; Canton, G.H.; Pitt, D. Black globe-humidity index (BGHI) as comfort equation for dairy cows. Transaction of the ASAE, St. Joseph, v.24, n.3, p.711-714, 1981.

Cochran, W.G.; Cox, G.M. Experimental designs. New York: John Wiley and Sons, 1957.661p.

Giovani, M. Response surface methodology and product optimization. Food Technology, London, v.37, n.11, p.41-83, 1983.

Henika, R.G. Simple and effective system for use with response surface methodology. Cereal Science Today, St. Paul, v.17, n.10, p.309-334, 1972.

Hevener, O.F. All about humiture. Weatherwise, Washington, v.12, n.5, p.83-85, 1959.

Houghten, F.C.; Yaglou, C.P. Determining lines of equal comfort. ASHVE Transaction, St. Joseph, v.29, n.4, p.163-176, 1923.
McArdle, B.; Dunham, W.; Holling, H.E.; Ladell, W.S.S; Scott, J.W.; Thomson, M.L.; Weiner, J.S. The prediction of the physiological effects of warm and hot environments: the P4SR index. London: Medical Research Council, 1947. 47p.

Medeiros, C.M. Ajuste de modelos e determinação de índice térmico ambiental de produtividade para frangos de corte: Viçosa: UFV, 2001. 125p. Tese Doutorado

Rosenberg, N.J.; Blad, B.L.; Verma, S.B. Microclimate: the biological environment. New York: Wiley-Interscience Publication, 1983. 495p.

Steadman, R.G. The assessment of sultriness. Part I: A temperature-humidity index based on human physiology and clothing science. Journal of Applied Meteorology, Boston, v. 18, n.7, p.861-873, 1979.

Thom, E.C. The discomfort index. Weatherwise, Washington, v.12, n.2, p.57-60, 1959. 